\title{
Fucoidan inhibits angiogenesis induced by multiple myeloma cells
}

\author{
FEN LIU $^{1 *}$, GUOPING LUO $^{1 *}$, QING XIAO $^{1}$, LIPING CHEN $^{1}$, \\ XIAOHUA LUO ${ }^{1}$, JINGLONG LV ${ }^{1}$ and LIXUE CHEN ${ }^{2}$ \\ ${ }^{1}$ Department of Hematology and ${ }^{2}$ The Central Laboratory, \\ The First Affiliated Hospital of Chongqing Medical University, Chongqing 400016, P.R. China
}

Received February 20, 2016; Accepted March 30, 2016

DOI: $10.3892 /$ or.2016.4987

\begin{abstract}
Multiple myeloma (MM) remains an incurable hematological neoplasms. Our previous studies showed that Fucoidan possessed anti-myeloma effect by inducing apoptosis and inhibiting invasion of myeloma cells. In this study, we evaluated the effect of Fucoidan on angiogenesis induced by human myeloma cells and elucidated its possible mechanisms. Multiple myeloma cells were treated with Fucoidan at different concentrations, then the conditioned medium (CM) was collected. The levels of VEGF in the CM were tested by ELISA. The results showed that Fucoidan significantly decreased VEGF secretion by RPMI-8226 and U266 cells. The tube formation assay and migration test on human umbilical vein endothelial cells (HUVECs) were used to examine the effect of Fucoidan on angiogenesis induced by human myeloma cells. The results showed that Fucoidan decreased HUVECs formed tube structures and inhibited HUVECs migration, and suppressed the angiogenic ability of multiple myeloma RPMI-8226 and U266 cells in a dose-dependent manner. The study also showed that Fucoidan downregulated the expression of several kinds of proteins, which may be correlated with the reduction of angiogenesis induced by myeloma cells. Moreover, results were compared from normoxic and hypoxic conditions, they showed that Fucoidan had anti-angiogenic activity. Furthermore, in a multiple myeloma xenograft mouse model, it indicated that Fucoidan negatively affected tumor growth and angiogenesis in vivo. In conclusion, our results demonstrate that Fucoidan was able to interfere with angiogenesis of multiple myeloma cells both in vitro and in vivo and may have a substantial potential in the treatment of MM.
\end{abstract}

Correspondence to: Dr Qing Xiao, Department of Hematology, The First Affiliated Hospital of Chongqing Medical University, 1 Youyi Road, Yuzhong, Chongqing 400016, P.R. China E-mail: 13220327680@163.com

${ }^{*}$ Contributed equally

Key words: multiple myeloma, human umbilical vein endothelial cells, Fucoidan, angiogenesis, hypoxia, mechanisms

\section{Introduction}

Multiple myeloma (MM), the second most common hematological malignancy following non-Hodgkin's lymphoma (NHL), contribute to $13 \%$ of all malignancies and $1 \%$ of all neoplasias (1). MM is characterized by bone lesions, anemia, susceptibility to infections, renal failure and hypercalcemia, caused by proliferation of clonal plasma cells in the bone marrow (2). Recently, some agents such as thalidomide, lenalidomide, and bortezomib have prolonged overall survival of MM patients, but MM remains an incurable disease and eventually almost all patients relapse and become resistant to the chemical treatment. Therefore, there is an urgent need to explore new therapeutic agent to improve the survival of MM patients.

In MM, angiogenesis takes place in the microenvironment and that is strongly correlated to disease progression and poor prognosis (3). Hypoxia inducible factor $1 \alpha(\mathrm{HIF}-1 \alpha)$ and vascular endothelial growth factor (VEGF) are known to play important roles in angiogenesis and tumor progression (4). It is hypoxic in the bone marrow microenvironment of MM patients (5), and HIF-1 $\alpha$ has been regarded as the most important factor promoting angiogenesis by upregulating pro-angiogenic factors such as VEGF (6). In MM, VEGF is secreted not only by myeloma cells but also bone marrow stem cells (BMSC) (7). The activation of extracellular signal-regulated kinases $1 / 2$ (ERK1/2) and phosphatidylinositol 3-kinase/protein kinase B (PI3K/AKT) pathway are critical for myeloma cells to express HIF-1 $\alpha$ and VEGF (8).

Fucoidan is a kind of marine drug, and its anticancer function has been focused on. It is clear that Fucoidan has potential to inhibit the proliferation of cancer cells (9-11). Furthemore, Fucoidan suppressed neovascularization of human umbilical vein endothelial cells (HUVECs) in vitro and tumor-bearing animal models $(9,10,12,13)$. Previously, we found that Fucoidan was able to reduce MM cell escape caused by various chemotherapy drugs, which might prevent the formation of MRD (minimal residual desease) and occurrence of relapse (14). However, whether Fucoidan has the ability to suppress HIF-1 $\alpha /$ VEGF expression in human multiple myeloma cells still remains unknown. Thus, in this study, we investigated whether Fucoidan can significantly inhibit angiogenesis of MM both in MM cells and a xenograft mouse model. 


\section{Materials and methods}

Reagents. Fucoidan was purchased from Sigma-Aldrich (St. Louis, MO, USA), and dissolved in PBS (Sigma) and sterilized by a $0.22-\mathrm{mm}$ syringe filter (Millipore, Carrigtwohill, Ireland). Total and phospho-specific antibodies against AKT, and ERK1/2 were purchased from Cell Signaling Technology (Beverly, MA, USA). Antibodies against HIF-1 $\alpha$ and VEGF were obtained from Abcam (Cambridge, UK).

Animals. Four to six-week-old female severe combined immunodeficiency/non-obese diabetic (NOD/SCID) mice (HFK Bioscience, Beijing, China), with body weight 15-18 g, were housed under individual ventilated cages (IVC) in a room maintained at constant temperature under 12-h light and darkness cycle at the Laboratory Animal Center of Chongqing Medical University. All procedures associated with animals was reviewed and approved by the Institutional Animal Care and Use Committee of Chongqing Medical University.

Cell culture and induction of hypoxia. Human MM cell lines (RPMI-8226 and U266) were kept frozen in our laboratory and human umbilical vein endothelial cells (HUVECs) were kindly provided by Dr Qifu Li (Department of Geriatrics, The First Affiliated Hospital of Chongqing Medical University). All cell lines were maintained with RPMI-1640 (SigmaAldrich, St. Louis, MO, USA) containing 10\% fetal bovine serum (Gibco, Grand Island, NY, USA), penicillin (100 U/ml), and streptomycin $(100 \mu \mathrm{g} / \mathrm{ml})$ (Beyotime, Bejing, China) and subsequently incubated at $37^{\circ} \mathrm{C}$ in humid air with $19 \%$ $\mathrm{O}_{2}, 5 \% \mathrm{CO}_{2}$. For hypoxia induction, cells were incubated in a hypoxic chamber with a gas mixture $\left(94 \% \mathrm{~N}_{2}, 5 \% \mathrm{CO}_{2}\right.$ and $1 \% \mathrm{O}_{2}$ ) before being treated with Fucoidan.

Collection of conditioned medium (CM). Condition media (CM) was collected as described (15). Briefly, $5 \times 10^{5} / \mathrm{ml}$ RPMI-8226 and U266 cells were cultured with different concentrations of Fucoidan $(0,25,50,100$ and $200 \mu \mathrm{g} / \mathrm{ml})$ for $72 \mathrm{~h}$ under normoxic and hypoxic conditions. After treatment, cells were recollected and $\mathrm{CM}$ was collected, and centrifuged at $12,000 \mathrm{rpm}$ for $10 \mathrm{~min}$ under $4^{\circ} \mathrm{C}$. $\mathrm{CM}$ was either used immediately or stored at $-20^{\circ} \mathrm{C}$.

ELISA. CM of each group were collected as above, human VEGF ELISA kit (Boster, Wuhan, China) was used to measure the concentration of VEGF according to the manufacturer's protocol. Then the plate was read immediately at $450 \mathrm{~nm}$ on a microplate reader (Amersham Pharmacia Biotech, USA), and the concentration was determined by calculating formula from a standard curve. The experiment was performed three times.

Tube formation assay. The ability of HUVECs induced by CM was assessed by the tube formation assay. Matrigel $(50 \mu \mathrm{l} / \mathrm{well}$, BD Pharmingen, San Diego, CA, USA) was added into a 96-well plate and incubated for $30 \mathrm{~min}$ at $37^{\circ} \mathrm{C}$. HUVECs $\left(2 \times 10^{4}\right.$ cells/well $)$ were suspended in CM $(100 \mu \mathrm{l} /$ well $)$ and plated on top of Matrigel, then incubated for $8 \mathrm{~h}$ and the capillary-like structures were observed under a microscope. Three random microscopic fields were photographed to evaluate the capillary-like structure formation (x200), and the tube areas were quantified by using Image-Pro Plus software 5.0 (Media Cybernetics, MD, USA).

Transwell migration assay. The effect of Fucoidan on migration of HUVECs was demonstrated in 24-well Transwell cell culture chambers with the upper chamber containing filters. HUVECs $\left(2 \times 10^{4}\right)$ were plated in the upper chambers, followed by cocultivation with $50 \% \mathrm{CM}$ in the lower chamber. Incubating the plate for $6 \mathrm{~h}$ at $37^{\circ} \mathrm{C}$, media in the upper chamber were sucked out and cells on the surface of the upper chamber were removed gently with a cotton swab. Cells on the lower surface of the filters were fixed with $4 \%$ poly paraformaldehyde solution for $20 \mathrm{~min}$, stained with $0.1 \%$ crystal violet solution for $10 \mathrm{~min}$, then washed by PBS twice. Five random microscopic fields were photographed (x200), and the number of HUVECs were counted by using Image-Pro Plus software 5.0 (Media Cybernetics).

Western blot analysis. After being cultured with Fucoidan for $72 \mathrm{~h}$ under normoxic and hypoxic conditions, cells were collected and washed with ice-cold PBS twice. Cell extracts were prepared with cell lysis buffer mixed with protease and phosphatase inhibitors (KeyGen Biotech, Nanjing, China). Total protein concentration was determined using the BCA protein assay kit (Beyotime). Aliquots of proteins (60 $\mu \mathrm{g} / \mathrm{lane})$ were separated in $12 \%$ SDS-PAGE and transferred onto PVDF membrane (Bio-Rad, Hercules, CA, USA), then blocked with $5 \%$ non-fat milk for $2 \mathrm{~h}$. Membranes were incubated with primary antibodies overnight at $4^{\circ} \mathrm{C}$ as follows: HIF- $1 \alpha$ and VEGF (Abcam, dilution: 1:1,000 and 1:600), AKT, phosphoAKT, ERK 1/2, and phospho-ERK 1/2 (Cell Signaling Technology, dilution: 1:1,000), and $\beta$-actin (Boster, dilution: $1: 2,000)$ was used as the internal control. After washing with TBS-Tween-20, the membranes were incubated with horseradish peroxidase (HRP)-conjugated goat anti-rabbit or mouse IgG as the secondary antibody (Boster, dilution: 1:2,000) for $2 \mathrm{~h}$ at $37^{\circ} \mathrm{C}$. The protein bands were visualized using an enhanced chemiluminescence (ECL, Beyotime) kit according to the manufacturer's instructions.

Mouse xenograft model. The NOD/SCID mice were injected subcutaneously with $1 \times 10^{7}$ RPMI-8226 cells which were suspended in $100 \mu 1$ of serum-free RPMI-1640 medium, together with $100 \mu \mathrm{l}$ Matrigel in the right flank (16). To prevent leakage, a cotton swab was held cautiously for 1 min over the site of injection. The weight of the mice and tumor size were measured and recorded. Tumors were measured with vernier caliper every other day and volumes were calculated according to the formula: $\mathrm{V}=a^{2} b / 2$, where $a$ is the major axis and $b$ is the minor axis. When the volume was measurable, the mice ( $n=6 /$ group) were assigned to three groups randomly, group I as the control one treated with same volume of PBS, groups II and III treated by intraperitoneal injection with 10, $50 \mathrm{mg} / \mathrm{kg}$ Fucoidan every two days. After 3 weeks of treatments, the mice were sacrificed by cervical dislocation and the tumors were removed and measured. Fixing the tumors with $4 \%$ poly paraformaldehyde solution for $48 \mathrm{~h}$, embedded in paraffin, sectioned at $4 \mu \mathrm{m}$, and stained with hematoxylin and eosin (H\&E). 

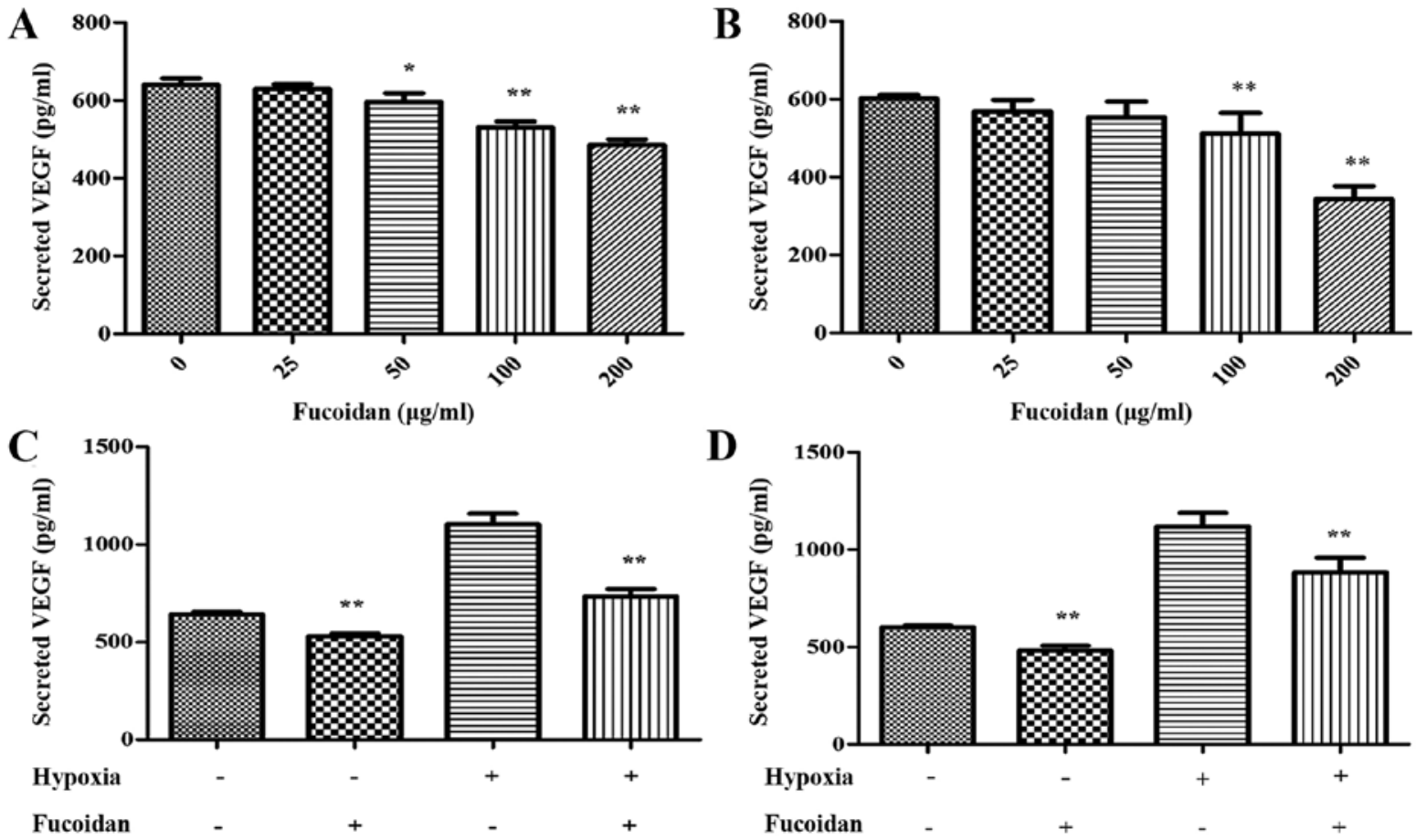

Figure 1. Fucoidan suppressed secretion of VEGF in myeloma cells. (A and B) RPMI-8226 and U266 cells were treated by Fucoidan for $72 \mathrm{~h}$ under normoxic condition. VEGF secretion was detected by ELISA assay. The secretion of VEGF was decreased dose-dependently in RPMI-8226 and U266 cells. Bars were the mean $\pm \mathrm{SD}(\mathrm{n}=3)$. The comparisons were made relative to untreated controls, and the different levels of significance are indicated as ${ }^{*} \mathrm{P}<0.05$ and ${ }^{* *} \mathrm{P}<0.01$. (C and D) RPMI-8226 and U266 cells were treated by Fucoidan for $72 \mathrm{~h}$ under normoxic and hypoxic condition. The secretion of VEGF was increased under hypoxia condition, and Fucoidan $(100 \mu \mathrm{g} / \mathrm{ml})$ can play a role also in inhibition. Bars are the mean $\pm \mathrm{SD}(\mathrm{n}=3)$. The comparisons were made relative to untreated controls, and the different levels of significance are indicated as ${ }^{*} \mathrm{P}<0.05$ and ${ }^{* *} \mathrm{P}<0.01$.

TUNEL assay for apoptotic cells in vivo. Apoptosis of tumor tissue sections were detected using terminal deoxynucleotidyl transferase (TdT)-mediated dUTP-digoxigenin nick-end labeling (TUNEL) assays with a TUNEL apoptosis assay kit (Beyotime). Tumor tissue sections were dewaxed, rehydrated through graded alcohols to water. Then, the sections were incubated with $3 \% \mathrm{H}_{2} \mathrm{O}_{2}$ for $10 \mathrm{~min}$ at $37^{\circ} \mathrm{C}$ and washed with PBS three times. The sections were blocked with goat serum for $10 \mathrm{~min}$. TUNEL assays were then performed according to the manufacturer's instructions.

Immunohistochemistry. For microvessel density (MVD) assay, immunohistochemical staining was performed by using IHC kit (Zhongshan Gold Bridge Bio, Beijing, China) according to the manufacturer's protocol. After the sections were dewaxed and antigen were repaired, the sections were incubated with Primary rabbit antihuman CD34 antibody (Zhongshan Gold Bridge Bio, dilution: 1:150) overnight at $4^{\circ} \mathrm{C}$. Then the sections were stained with a streptavidin-peroxidase system, the signal was visualized by using 3, 3'-diaminobenzidine (DAB) and counterstaining was done with hematoxylin. Areas of neovascularization were counted at high power magnification (x400) by two investigators in a blinded manner using Image-Pro Plus 5.0 (Media Cybernetics).

Statistical analysis. The data was expressed as mean \pm SD of a representative experiment in triplicate. Data were analyzed using One-way ANOVA and Student's t-test. The level of significance was indicated as $\mathrm{P}<0.05$ and $\mathrm{P}<0.01$.

\section{Results}

Fucoidan suppresses secretion of VEGF in myeloma cells. The VEGF secreted by myeloma cells is essential to angiogenesis, so we quantified the concentration of VEGF in the CM by ELISA assay. Under normoxic condition, the secretion of VEGF was decreased dose-dependently (Fig. 1A and B). As shown in Fig. 1C and D, it was obvious that the secretion of VEGF was increased under hypoxia condition, at the same time, Fucoidan at $100 \mu \mathrm{g} / \mathrm{ml}$ could lead to the suppression of VEGF secrete apparently in both myeloma cell lines.

Fucoidan decreases tube formation of HUVECs in vitro. To investigate the effect of Fucoidan on HUVECs in vitro, tube formation assay was conducted. HUVECs resuspended in the $\mathrm{CM}$ of multiple myeloma cells were seeded onto Matrigel and incubated for $6 \mathrm{~h}$. HUVECs spread and formed capillary-like structures. Compared with the control group, number and tube size of capillary structures stimulated by CM from myeloma cells pretreated with Fucoidan was reduced, the tubes were thinner and highly disconnected $(\mathrm{P}<0.01)$ (Fig. 2).

When HUVECs pretreated with CM from hypoxic condition, the HUVECs spread and formed tubes better than that with $\mathrm{CM}$ from normoxic condition $(\mathrm{P}<0.01)$ (Fig. 3).

Fucoidan inhibits migration of HUVECs cocultured with CM of multiple myeloma cells. To assess the potential effect of Fucoidan in HUVECs migration, a Transwell migration assay was performed. After treated with $\mathrm{CM}$ from normoxic 

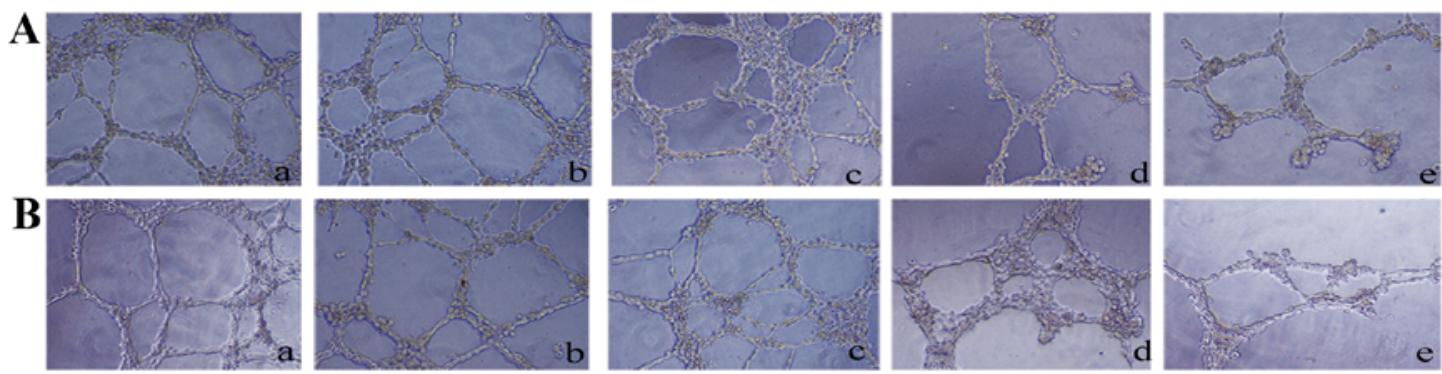

C
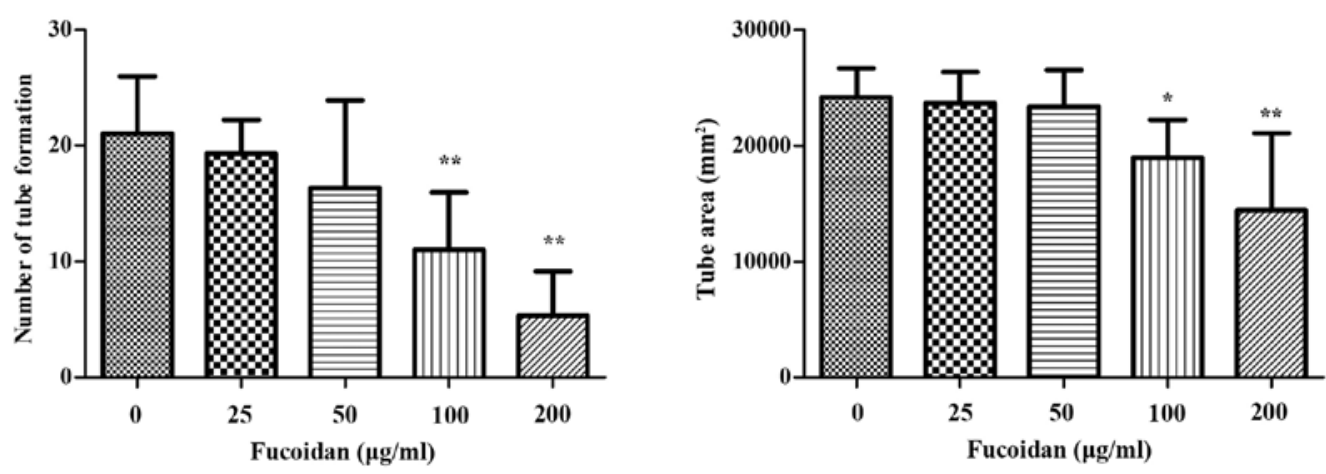

D
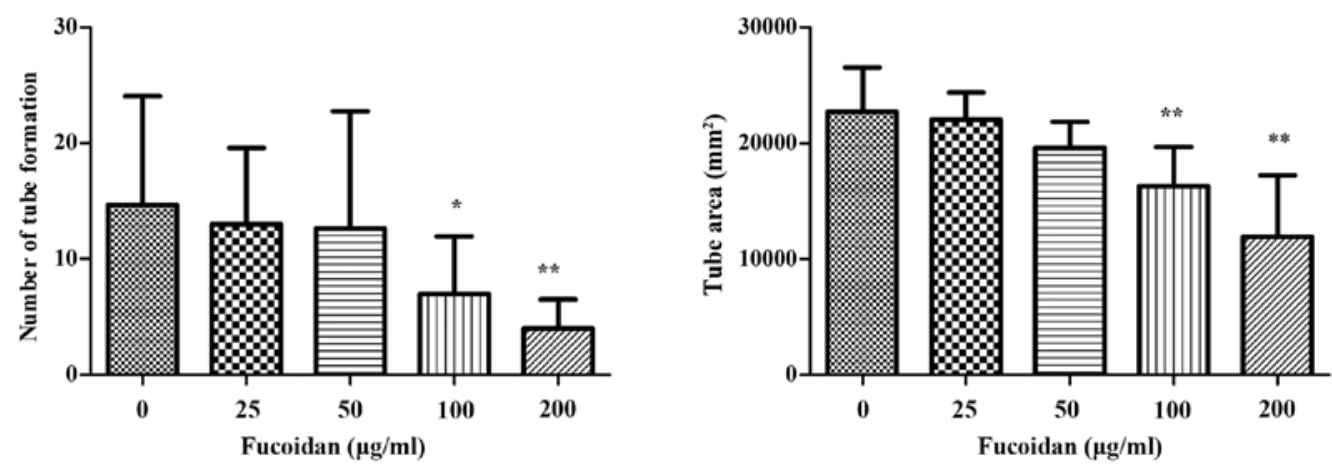

Figure 2. Pretreated with CM and then by Fucoidan inhibit the tube formation of HUVECs under normoxic condition. (A and B) Fucoidan inhibits HUVECs angiogenesis induced by CM from Fucoidan pretreated RPMI-8226 on the Matrigel in a dose-dependent manner. (C and D) The number and area of tubes were evaluated, they were inhibited significantly compared with the untreated controls. Bars are the mean $\pm \mathrm{SD}(\mathrm{n}=3)$ and the different levels of significance are indicated as ${ }^{*} \mathrm{P}<0.05$ and ${ }^{* *} \mathrm{P}<0.01$.

condition, the results indicated that less HUVECs migrated to the lower chamber when the myeloma cells were cultured with $\mathrm{CM}$ from the high dose-Fucoidan pretreated, similar results were obtained in both myeloma cells $(\mathrm{P}<0.01)$ (Fig. 4). CM from hypoxic condition promoted HUVECs migration, when pretreated with Fucoidan at $100 \mu \mathrm{g} / \mathrm{ml}$, HUVECs migration was significantly inhibited $(\mathrm{P}<0.01)$ (Fig. 5).

Effect of Fucoidan on the expression of various proteins involved in angiogenesis. After myeloma cells were treated with Fucoidan under normoxic condition, HIF-1 $\alpha$ and VEGF were inhibited when Fucoidan was increased. To ascertain which signaling pathways were involved in regulating VEGF secretion and expression of multiple myeloma cells by Fucoidan, we focused on the level of phosphorylation of AKT and ERK1/2. It was shown that the expression of p-AKT and p-ERK1/2 were significantly decreased when Fucoidan was increased (Fig. 6).

We further evaluated the effect by western blot analysis. The results showed that hypoxia induced higher levels of
HIF-1 $\alpha$ and VEGF in myeloma cells, and so did p-AKT and p-ERK1/2. Then, we determined whether Fucoidan reduces angiogenesis induced by myeloma cells. Treated with Fucoidan $100 \mu \mathrm{g} / \mathrm{ml}$, it is obvious that HIF- $1 \alpha$, VEGF and p-AKT were inhibited in both kinds of myeloma cells, but p-ERK1/2 changed little, as shown in Fig. 7.

Fucoidan markedly decreases tumor growth in a xenograft mouse model of human MM. We also assessed the efficacy of Fucoidan in vivo using a mouse model of human MM. NOD/SCID mice were given subcutaneous inoculations into the right flank with $1 \times 10^{7}$ RPMI- 8226 cells and then Fucoidan was given to mice by i.p. every two days. At the end of the treatment, the average tumor volume and weight in the Fucoidan treated group ( $50 \mathrm{mg} / \mathrm{kg}$ body weight) was significantly smaller and lighter than that in group I and II (Fig. 8A and $\mathrm{C}$ ). In addition, more necrotic tissues and disorderly and irregular tumor cell arrangements were observed in $\mathrm{H} \& \mathrm{E}$ stains of Fucoidan-treated mice, compared with untreated groups (Fig. 8B). TUNEL assay showed that the apoptotic 
A

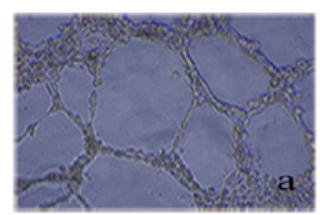

B

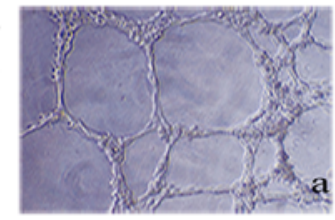

C

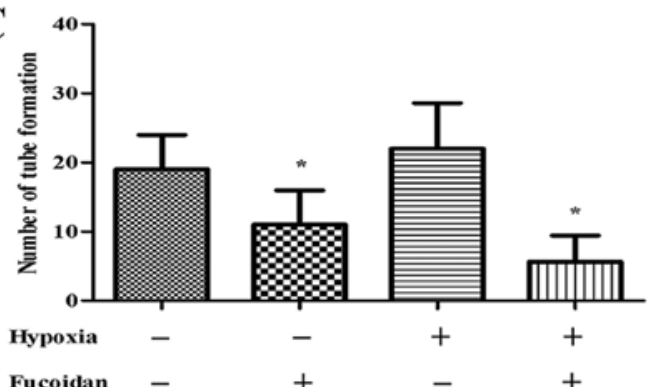

D

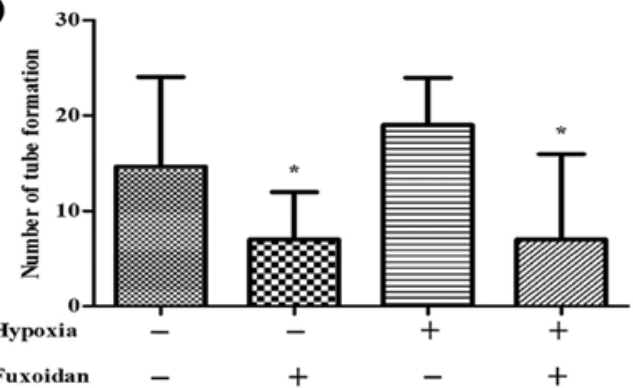

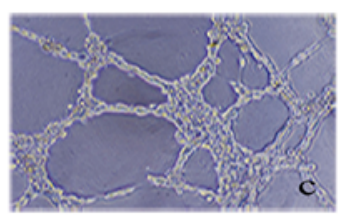
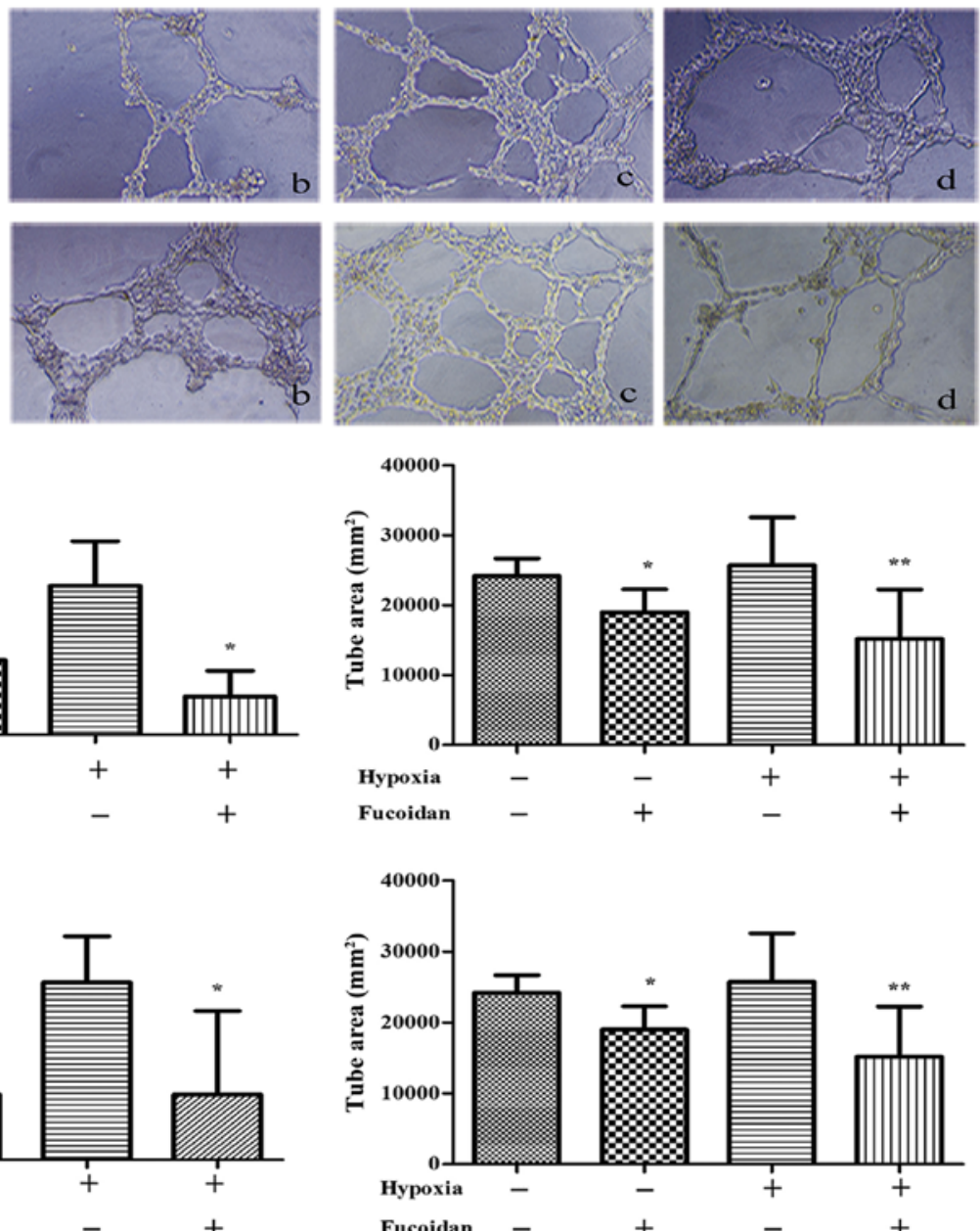

Figure 3. Pretreated with $\mathrm{CM}$ then by Fucoidan inhibit tube formation of HUVECs under hypoxic condition. (A and B) CM from hypoxic condition, enhanced the quantity of tubes formed by HUVECs. Fucoidan inhibits HUVEC angiogenesis induced by CM from Fucoidan $100 \mu \mathrm{g} / \mathrm{ml}$. (C and D) The number and area of tubes were evaluated, they were not increased significantly compared with the untreated controls, but when treated with Fucoidan, they were increased compared with the untreated controls. Bars are the mean $\pm \mathrm{SD}(\mathrm{n}=3)$ and the different levels of significance are indicated as ${ }^{*} \mathrm{P}<0.05$ and ${ }^{* *} \mathrm{P}<0.01$.
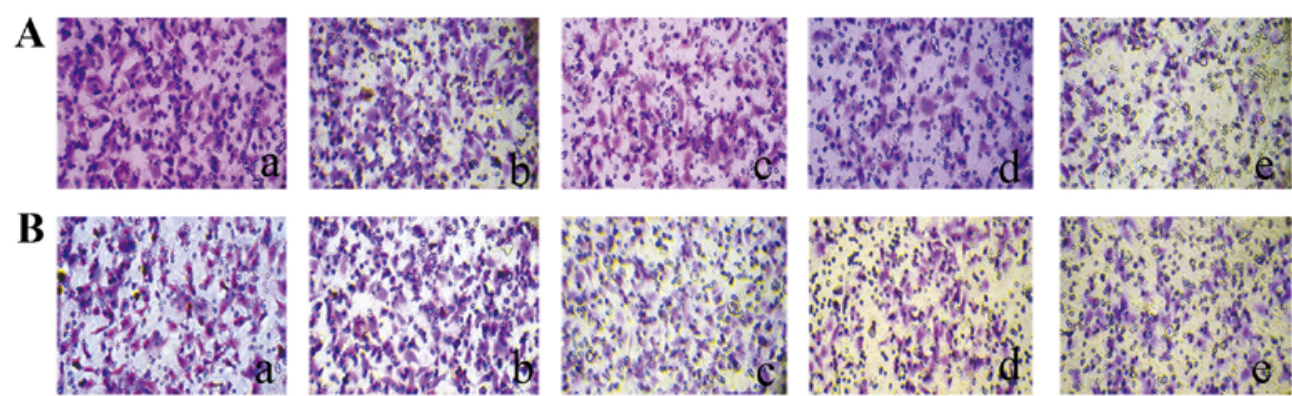

C
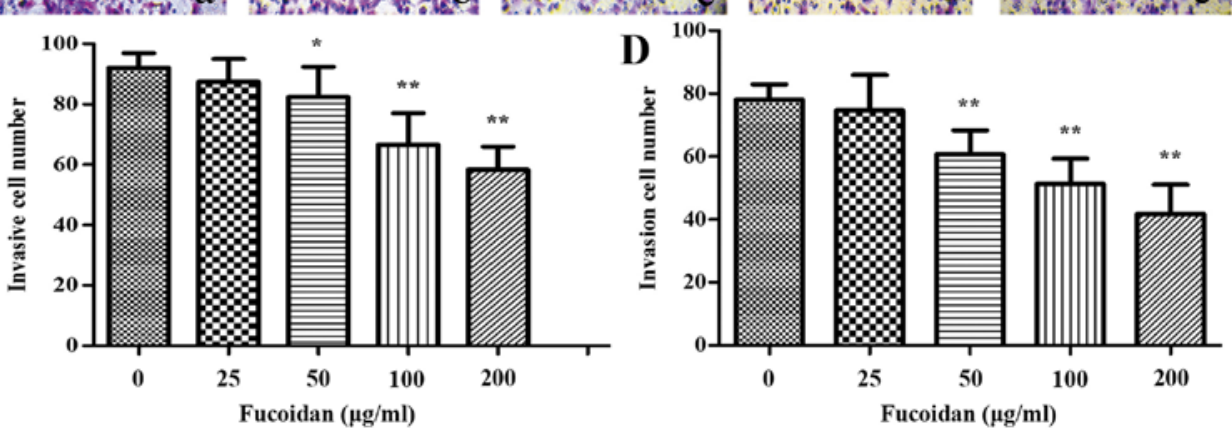

Figure 4. Fucoidan inhibits the migration of HUVECs under normoxic condition. (A and B) Transwell migration assay indicated that less HUVECs migrated when the cells were cultured with CM from the high dose-Fucoidan pretreated RPMI-8226 or U266. (C and D) The number of HUVECs was evaluated, they were inhibited significantly compared with the untreated controls. Bars are the mean $\pm \mathrm{SD}(\mathrm{n}=3)$ and the different levels of significance are indicated as ${ }^{*} \mathrm{P}<0.05$ and ${ }^{* *} \mathrm{P}<0.01$ 

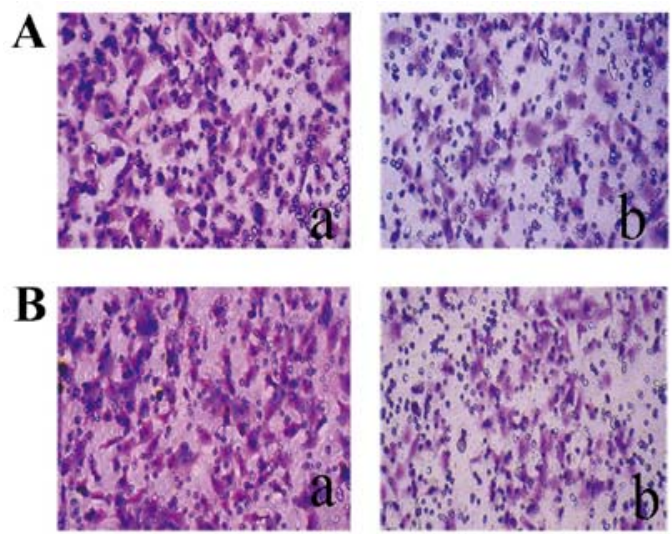

C

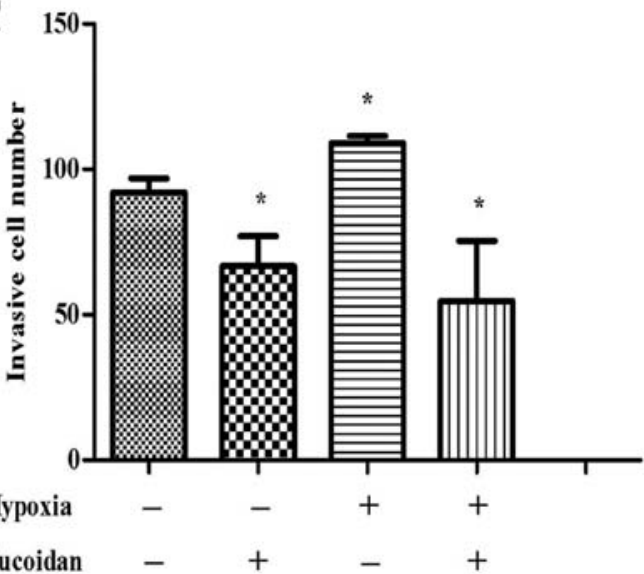

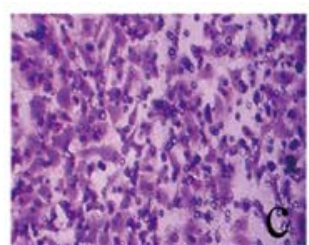
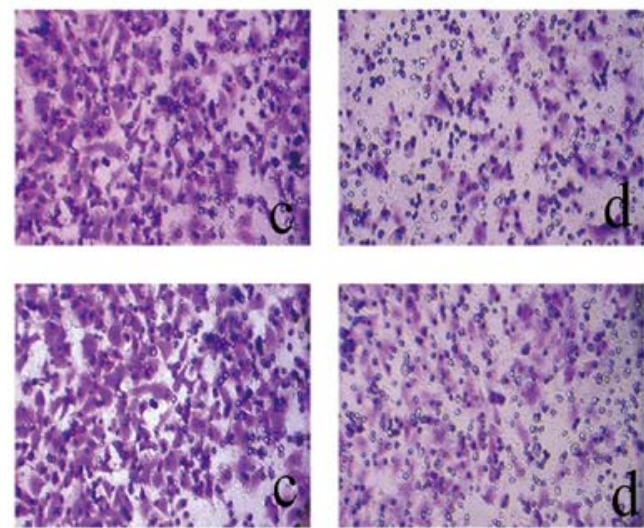

D

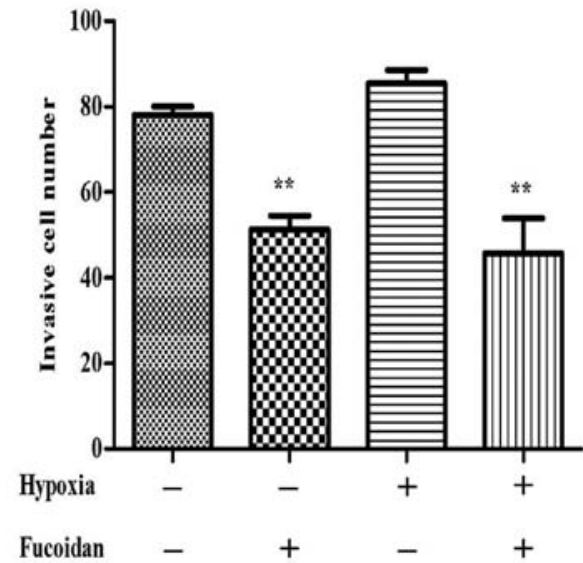

Figure 5. Fucoidan inhibits the migration of HUVECs under two conditions. (A and B) Transwell migration assay indicated that hypoxia induced greater HUVECs migration. (C and D) The number of HUVECs were evaluated, hypoxia induced more HUVEC migration and they were inhibited significantly treated with Fucoidan $100 \mu \mathrm{g} / \mathrm{ml}$ compared with the untreated controls. Bars are the mean $\pm \mathrm{SD}(\mathrm{n}=3)$ and the different levels of significance are indicated as ${ }^{*} \mathrm{P}<0.05$ and ${ }^{* *} \mathrm{P}<0.01$.

cells were increased significantly in treated mice (Fig. 8D). Immunohistochemistry for $\mathrm{CD} 34^{+}$on tumor sections showed a significant reduction in the number of $\mathrm{CD} 34^{+}$vessels within group III (median number of vessels positive for $\mathrm{CD} 34^{+}$per vision 8 vs $2, \mathrm{P}<0.05$ ) (Fig. 8E). Taken all together, these results demonstrated that Fucoidan inhibited tumor growth in vivo, induced MM-cell apoptosis, and decreased the number of MVD.

\section{Discussion}

Fucoidan, extracted from brown algae species, is a polysaccharide and consists of sulfated fucose residues (17). As a promising anticancer agent, it has been shown that Fucoidan could inhibit the growth of a wide variety of tumor cells, but it is still unclear whether Fucoidan has any impact on multiple myeloma-induced angiogenesis. Therefore, we investigated the effects of Fucoidan on the inducing capability of multiple myeloma cells.

Angiogenesis is a complex process and essential for cancer progression and metastasis. In multiple myeloma patients, microvessel density in the bone marrow was significantly higher (18). We directly investigated the tube formation and invasion of HUVECs. We found that the number and the area of tube were significantly decreased when myeloma cells were pre-treated with Fucoidan. Furthermore, we compared the tube area between $\mathrm{CM}$ collected under normoxia and hypoxia, regardless whether treated with Fucoidan or not. It showed that Fucoidan had the same efficacy even though the environment changed. During angiogenesis processes, migration is a key step for the formation of new blood vessels (19). We next performed Transwell chamber migration assay, the results displayed a similar tendency. That suggesting that the use of Fucoidan can interfere with neovascularization.

Bone marrow microenvironment is also hypoxic in many hematological malignancies, such as non-Hodgkin lymphoma (20), acute myeloid leukemia (21), chronic lymphocytic leukemia (22), and MM (23). The HIF-1 $\alpha$ /VEGF/VEGFreceptor pathway is upregulated in MM cases and linked with increased angiogenesis. HIF1 $\alpha$ is an important transcription factor directly regulating the expression of the VEGF gene (24). Previous studies have shown that HIF-1 $\alpha$ inhibition can block angiogenesis (25), but under the opposite assumption, it promotes (26). Therefore, HIF-1 $\alpha$ may be a target to control MM cell-derived angiogenesis. Here, our results suggested that Fucoidan inhibited the expression of HIF-1 $\alpha$ protein in a dose-dependent manner in RPMI-8226 and U266 cells under normoxia. Hypoxia induced HIF-1 $\alpha$ accumulation, at the same time, HIF-1 could be inhibited when treated with Fucoidan.

To further clarify the mechanism of Fucoidan inhibiting angiogenesis induced by myeloma cells, the expression of VEGF protein was detected. Results showed that VEGF protein expression decreased with the treatment of increasing 
A

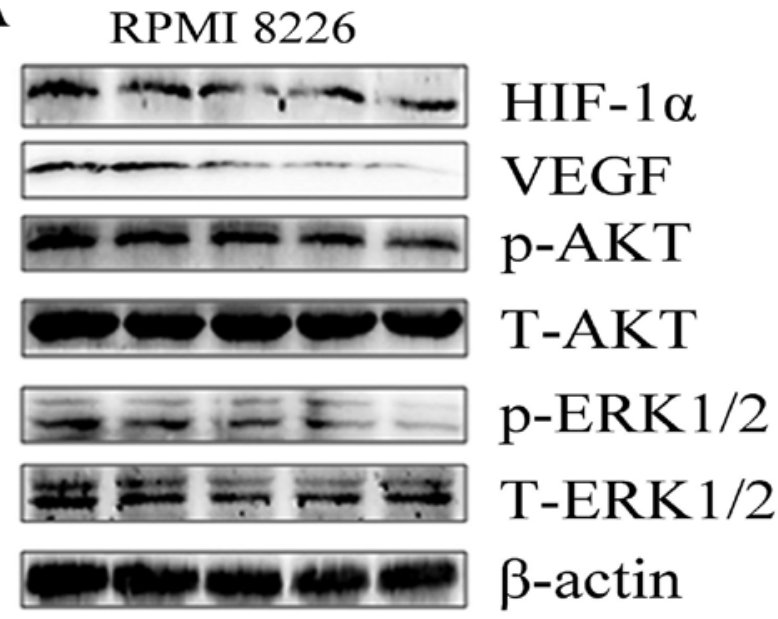

U266

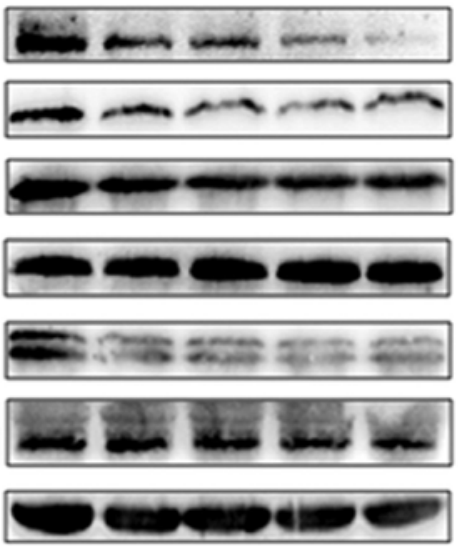

B

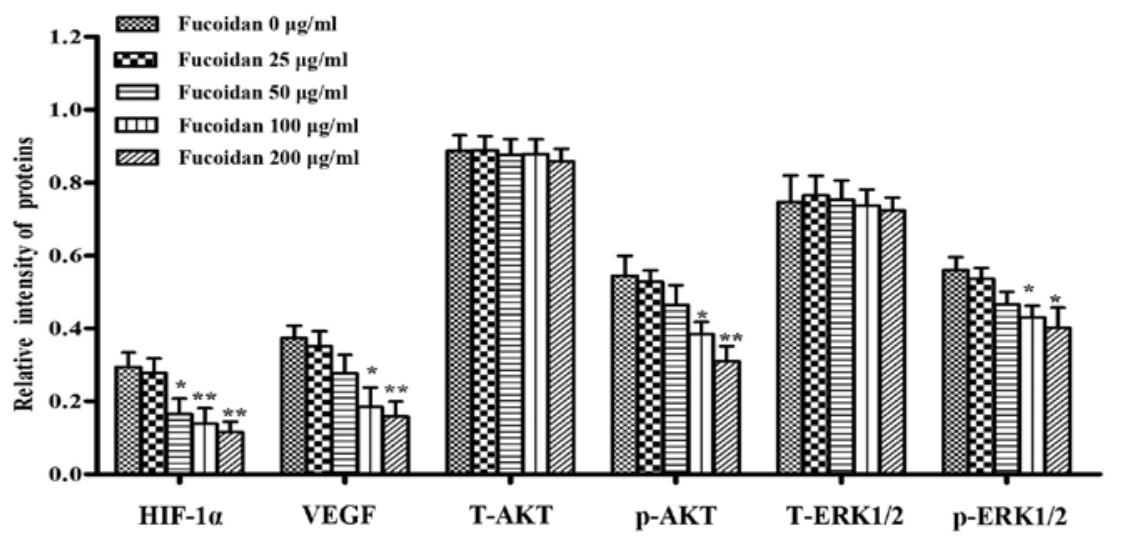

C

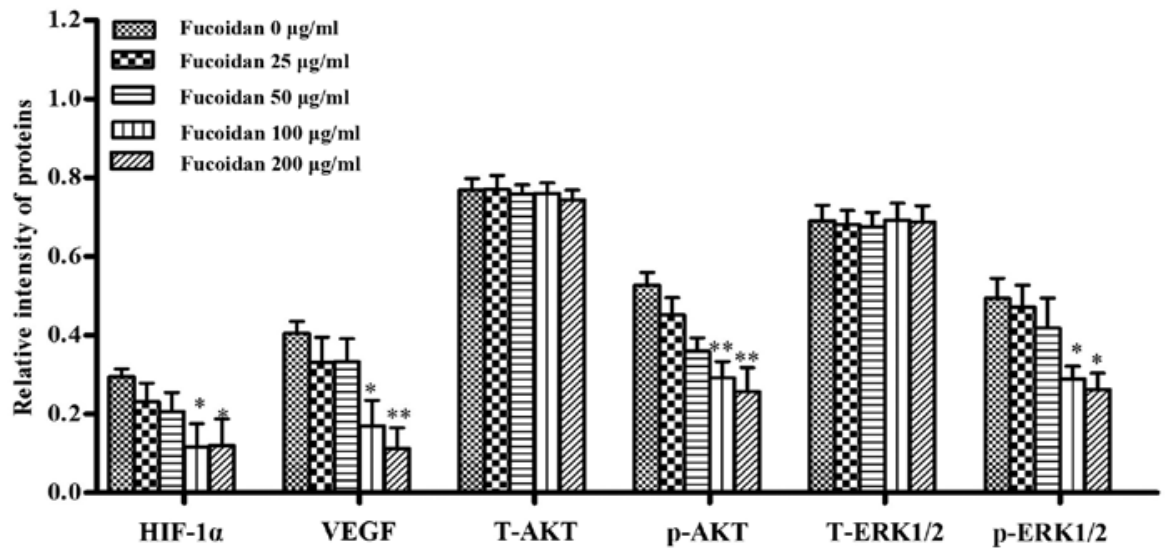

Figure 6. Fucoidan affects the expression of various proteins involved in angiogenesis under normoxic condition. (A) RPMI-8226 and U266 cells were treated with Fucoidan under normoxic condition, HIF- $1 \alpha$ and VEGF, p-AKT and p-ERK1/2 were inhibited when Fucoidan increase. (B) For quantity of RPMI-8226 cells, bars are the mean $\pm \mathrm{SD}(\mathrm{n}=3)$. The comparisons were made relative to $\beta$-actin, and the different levels of significance are indicated as ${ }^{*} \mathrm{P}<0.05$, ${ }^{* *} \mathrm{P}<0.01$. (C) For quantity of U266 cells, bars are the mean $\pm \mathrm{SD}(\mathrm{n}=3)$. The comparisons were made relative to $\beta$-actin, and the different levels of significance are indicated as ${ }^{*} \mathrm{P}<0.05,{ }^{* * *} \mathrm{P}<0.01$.

Fucoidan, and angiogenesis decreased accordingly. Hypoxia can stimulate the activation of PI3K/AKT pathway, the increase of HIF-1 $\alpha$ synthesis is associated with activated PI3K/AKT signaling. ERK can be activated by hypoxia and may be involved in the response to hypoxia (27). It should be considered that the possible crosstalk among these pathways, AKT and ERK pathways activated by VEGF and VEGF secretion can be reduced by inhibition of AKT or ERK protein kinase activity $(7,28)$. In multiple myeloma, downregulation of p-ERK1/2 activity reduces myeloma-induced angiogenesis by inhibiting VEGF secretion (29). Our results showed that p-AKT and p-ERK1/2 were inactivated in a dose-dependent manner under normoxia. Under hypoxia, p-AKT was activated and also, Fucoidan had the capability to inhibit it. Whereas p-ERK1/2 was not apparently activated, there was no sign that $\mathrm{p}-\mathrm{ERK} 1 / 2$ was restrained even treated with Fucoidan. It was not possible to tell whether PI3K/AKT pathway was more important than ERK1/2. 
A
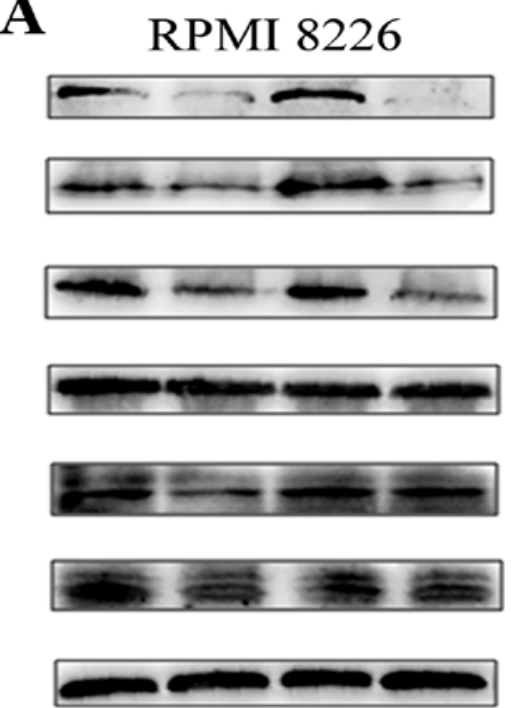

B

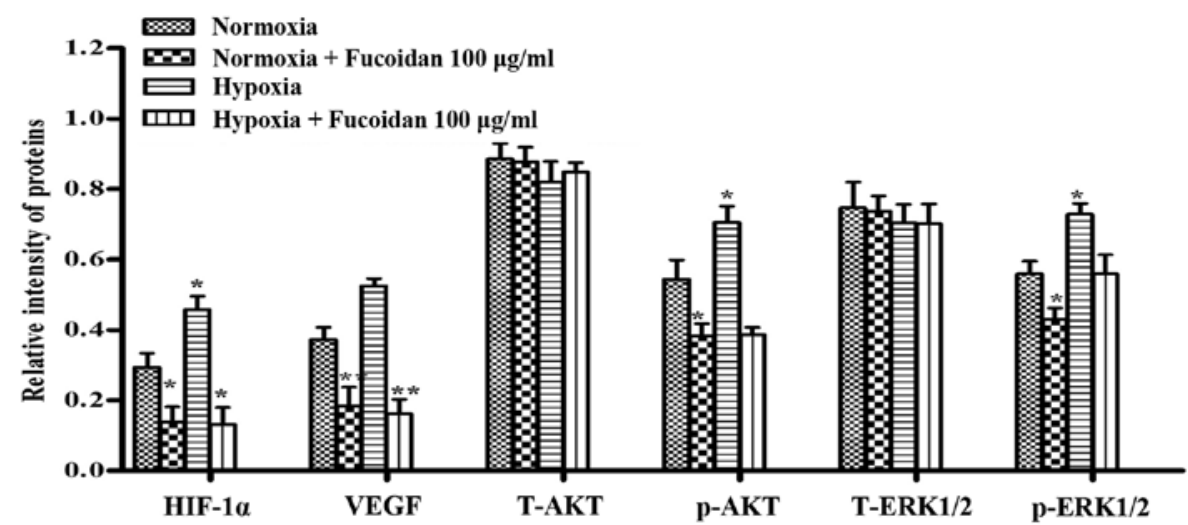

C

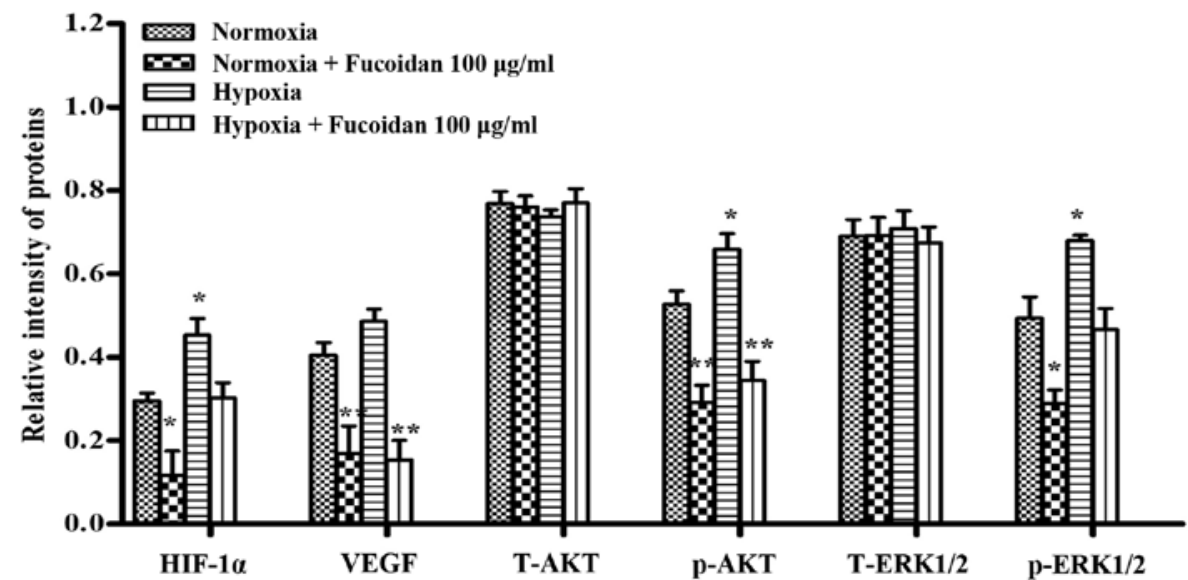

Figure 7. Fucoidan affected the expression of various proteins involved in angiogenesis under two conditions. (A) RPMI-8226 and U266 cells were treated with Fucoidan under normoxic and hypoxic conditions. (B) For quantity of RPMI-8226 cells, it showed that the expressions of HIF-1 $\alpha$, VEGF, p-AKT and p-ERK1/2 were increased without pretreatment, but not when pretreated with Fucoidan at $100 \mu \mathrm{g} / \mathrm{ml}$; VEGF and p-AKT expression increased under hypoxic condition, and the treatment of Fucoidan at $100 \mu \mathrm{g} / \mathrm{ml}$ inhibited VEGF, p-AKT, but not HIF-1 $\alpha$ and p-ERK1/2. Bars are the mean \pm SD ( $\mathrm{n}=3$ ). The comparisons were made relative to $\beta$-actin, and the different levels of significance are indicated as ${ }^{*} \mathrm{P}<0.05,{ }^{* *} \mathrm{P}<0.01$. (C) For quantity of $\mathrm{U} 266$ cells, it showed that the expressions of HIF-1 $\alpha$, p-AKT and p-ERK1/2 were increased without pretreatment, but not when pretreated with Fucoidan at $100 \mu \mathrm{g} / \mathrm{ml}$; VEGF and p-AKT expression increased under hypoxic condition, and the treatment of Fucoidan at $100 \mu \mathrm{g} / \mathrm{ml}$ inhibited VEGF, p-AKT, but not HIF-1 $\alpha$ and $\mathrm{p}-\mathrm{ERK} 1 / 2$. Bars are the mean $\pm \mathrm{SD}(\mathrm{n}=3)$. The comparisons were made relative to untreated controls, and the different levels of significance are indicated as ${ }^{*} \mathrm{P}<0.05,{ }^{* * *} \mathrm{P}<0.01$

A xenograft myeloma tumor model in NOD/SCID mice was set up. As the result show, consecutive administration of Fucoidan for 21 days significantly reduced the tumor volume and weight. In H\&E and TUNEL staining, the tumor cells were induced to apoptosis. In addition, CD34+ MVD expression in tumor sections were decreased. This confirms that tumor neovascularization was inhibited by Fucoidan, making the findings in vitro more certain. 

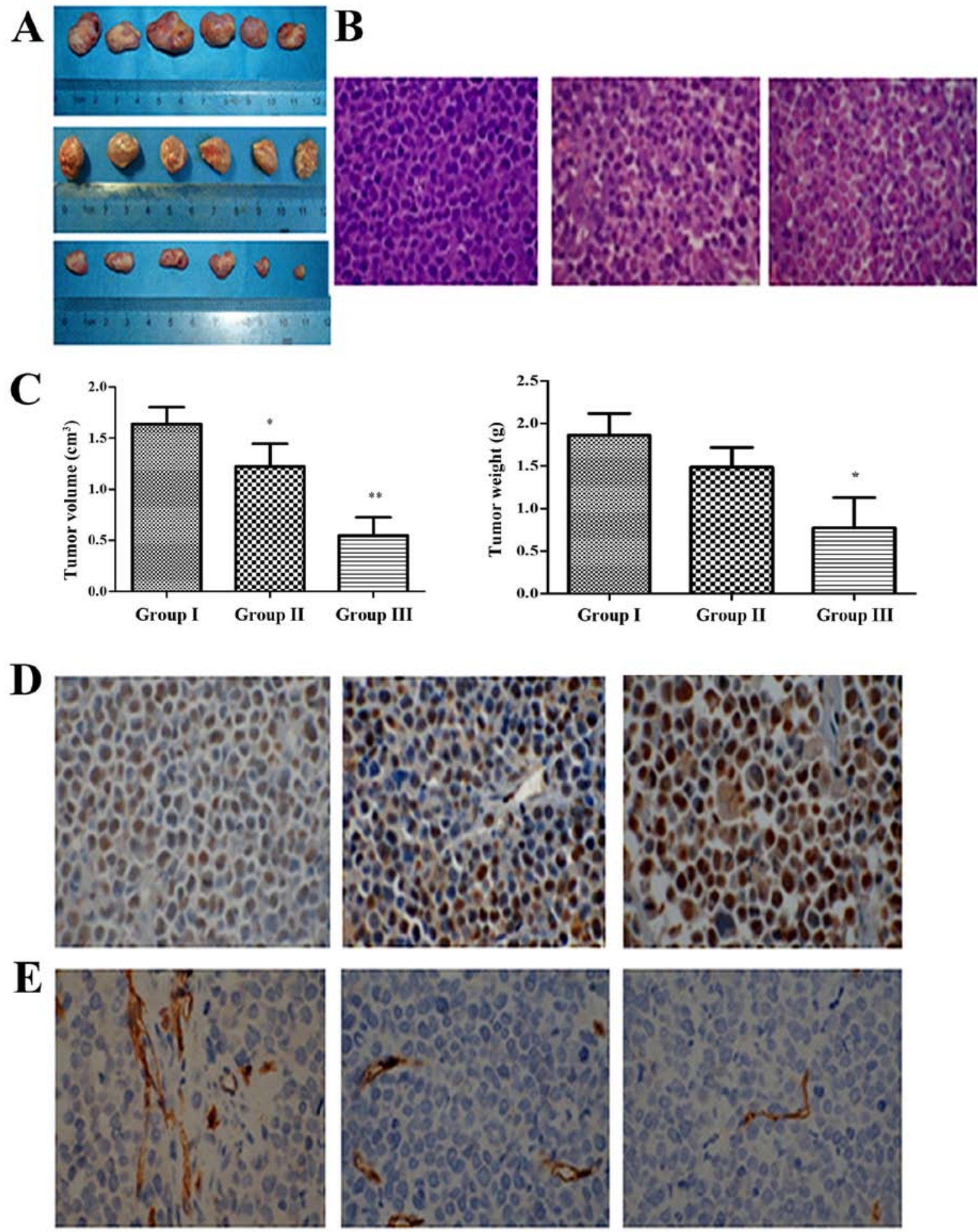

Figure 8. Fucoidan interfered with tumor growth in xenograft mouse model. (A) Multiple myeloma cells xenograft mouse model was built ( $\mathrm{n}=6$ ), group I was given PBS (100 $\mu$ L, i.p., every two days), group II and III were given Fucoidan (10 and $50 \mathrm{mg} / \mathrm{kg}$ body weight, i.p., every two days) for 3 weeks. Subcutaneous tumor tissue removed from the mice after the treatment. (B) H\&E staining showed that more disorder and irregular tumor cells arrangement in group III (x400). (C) Tumor volume and weight in mice were measured $(\mathrm{n}=6)$. Columns, mean; bars, $\mathrm{SD},{ }^{*} \mathrm{p}<0.05,{ }^{* *} \mathrm{p}<0.01$. (D) TUNEL assay showed that cell apoptosis rate was increased significantly by treatment with Fucoidan (x400). (E) MVD assay showed that the number of CD34 ${ }^{+}$vessels within mice of group III were decreased significantly $(x 400)$.

In conclusion, these results demonstrate that Fucoidan can prevent angiogenesis induced by myeloma cells. It may be related to the ability to downregulate HIF-1 $\alpha /$ VEGF protein levels under normoxia and hypoxia. The inhibition of HIF-1 $\alpha /$ VEGF protein expression is possibly associated with the suppression of PI3K/AKT pathway in both conditions, but it is remarkable that p-ERK1/2 is not obviously attenuated under hypoxia. Taken together, this study implies that Fucoidan might be a new potential agent for human multiple myeloma therapy. 


\section{Acknowledgements}

We would like to thank Dr Weixue Tang, Ms. Xiaoju Wang and Mr. Jie Liu for their excellent technical assistance, Dr Qifu Li (Department of Geriatrics, The First Affiliated Hospital of Chongqing Medical University) for HUVEC cells. This study was done in the First Affiliated Hospital of Chongqing Medical University Central Lab. This study was supported by the Key Subject of Chongqing Public Health Bureau (grant no. 2013-1-013).

\section{References}

1. Siegel R, Naishadham D and Jemal A: Cancer statistics, 2013. CA Cancer J Clin 63: 11-30, 2013.

2. Röllig C, Knop S and Bornhäuser M: Multiple myeloma. Lancet 385: 2197-2208, 2015

3. Ribatti D, Mangialardi G and Vacca A: Antiangiogenic therapeutic approaches in multiple myeloma. Curr Cancer Drug Targets 12: 768-775, 2012.

4. Gacche RN and Meshram RJ: Targeting tumor micro-environment for design and development of novel anti-angiogenic agents arresting tumor growth. Prog Biophys Mol Biol 113: 333-354, 2013.

5. Azab AK, Hu J, Quang P, Azab F, Pitsillides C, Awwad R, Thompson B, Maiso P, Sun JD, Hart CP, et al: Hypoxia promotes dissemination of multiple myeloma through acquisition of epithelial to mesenchymal transition-like features. Blood 119: 5782-5794, 2012.

6. Giatromanolaki A, Bai M, Margaritis D, Bourantas KL, Koukourakis MI, Sivridis E and Gatter KC: Hypoxia and activated $\mathrm{VEGF} /$ receptor pathway in multiple myeloma. Anticancer Res 30: 2831-2836, 2010.

7. Medinger $\mathrm{M}$, Fischer $\mathrm{N}$ and Tzankov A: Vascular endothelial growth factor-related pathways in hemato-lymphoid malignancies. J Oncol 2010: 729725, 2010.

8. Yang XM, Wang YS, Zhang J, Li Y, Xu JF, Zhu J, Zhao W, Chu DK and Wiedemann P: Role of PI3K/Akt and MEK/ERK in mediating hypoxia-induced expression of HIF-1alpha and VEGF in laser-induced rat choroidal neovascularization. Invest Ophthalmol Vis Sci 50: 1873-1879, 2009.

9. Han YS, Lee JH and Lee SH: Antitumor effects of Fucoidan on human colon cancer cells via activation of Akt signaling. Biomol Ther (Seoul) 23: 225-232, 2015.

10. Delma CR, Somasundaram ST, Srinivasan GP, Khursheed M, Bashyam MD and Aravindan N: Fucoidan from Turbinaria conoides: A multifaceted 'deliverable' to combat pancreatic cancer progression. Int J Biol Macromol 74: 447-457, 2015.

11. Yoshimoto M, Higaki K, Nanba E and Ikeguchi M: Antiproliferation activity of Fucoidan in MKN45 gastric cancer cells and downregulation of phosphorylated ASK1, a cell cycleregulated kinase. Yonago Acta Med 58: 1-7, 2015.

12. Zhu C, Cao R, Zhang SX, Man YN and Wu XZ: Fucoidan inhibits the growth of hepatocellular carcinoma independent of angiogenesis. Evid Based Complement Alternat Med 2013 692549, 2013.

13. Xue M, Ge Y, Zhang J, Wang Q, Hou L, Liu Y, Sun L and Li Q Anticancer properties and mechanisms of fucoidan on mouse breast cancer in vitro and in vivo. PLoS One 7: e43483, 2012.
14. Lv J, Xiao Q, Wang L, Liu X, Wang X, Yang Z, Zhang H and Dong P: Fucoidan prevents multiple myeloma cell escape from chemotherapy-induced drug cytotoxicity. Fitoterapia 84: 257-263, 2013.

15. Yang YC, Chen PN, Wang SY, Liao CY, Lin YY, Sun SR, Chiu CL, Hsieh YS, Shieh JC and Chang JT: The differential roles of Slit2-exon 15 splicing variants in angiogenesis and HUVEC permeability. Angiogenesis 18: 301-312, 2015.

16. Podar K, Raab MS, Zhang J, McMillin D, Breitkreutz I, Tai YT, Lin BK, Munshi N, Hideshima T, Chauhan D, et al: Targeting PKC in multiple myeloma: In vitro and in vivo effects of the novel, orally available small-molecule inhibitor enzastaurin (LY317615.HCl). Blood 109: 1669-1677, 2007.

17. Kwak JY: Fucoidan as a marine anticancer agent in preclinical development. Mar Drugs 12: 851-870, 2014.

18. Chen H, Shi L, Yang X, Li S, Guo X and Pan L: Artesunate inhibiting angiogenesis induced by human myeloma RPMI8226 cells. Int J Hematol 92: 587-597, 2010.

19. Masiero M, Simões FC, Han HD, Snell C, Peterkin T, Bridges E, Mangala LS, Wu SY, Pradeep S, Li D, et al: A core human primary tumor angiogenesis signature identifies the endothelial orphan receptor ELTD1 as a key regulator of angiogenesis. Cancer Cell 24: 229-241, 2013.

20. Minoia C, Quero C, Asselti M, Galise I, Marzano AL, Iacobazzi A, Rana A, Merchionne F, Serratì S, De Tullio G, et al: Changes in angiogenesis and hypoxia-inducible factor-1 $\alpha$ protein expression in relapsed/refractory indolent non-Hodgkin lymphomas. Br J Haematol 163: 640-645, 2013.

21. Drolle H, Wagner M, Vasold J, Kütt A, Deniffel C, Sotlar K, Sironi S, Herold T, Rieger C and Fiegl M: Hypoxia regulates proliferation of acute myeloid leukemia and sensitivity against chemotherapy. Leuk Res 39: 779-785, 2015.

22. Huelsemann MF, Patz M, Beckmann L, Brinkmann K, Otto T, Fandrey J, Becker HJ, Theurich S, von Bergwelt-Baildon M, Pallasch CP, et al: Hypoxia-induced p38 MAPK activation reduces Mcl-1 expression and facilitates sensitivity towards BH3 mimetics in chronic lymphocytic leukemia. Leukemia 29: 981-984, 2015.

23. Muz B, de la Puente P, Azab F, Luderer M and Azab AK: Hypoxia promotes stem cell-like phenotype in multiple myeloma cells. Blood Cancer J 4: e262, 2014.

24. Hashimoto T and Shibasaki F: Hypoxia-inducible factor as an angiogenic master switch. Front Pediatr 3: 33, 2015.

25. Storti P, Bolzoni M, Donofrio G, Airoldi I, Guasco D, Toscani D, Martella E, Lazzaretti M, Mancini C, Agnelli L, et al: Hypoxiainducible factor (HIF)- $1 \alpha$ suppression in myeloma cells blocks tumoral growth in vivo inhibiting angiogenesis and bone destruction. Leukemia 27: 1697-1706, 2013.

26. Fan GC: Hypoxic exosomes promote angiogenesis. Blood 124: 3669-3670, 2014

27. Chen TL, Zhu GL, Wang JA, Wang Y, He XL and Jiang J: Apoptosis of bone marrow mesenchymal stem cells caused by hypoxia/reoxygenation via multiple pathways. Int J Clin Exp Med 7: 4686-4697, 2014.

28. Giuliani N, Lunghi P, Morandi F, Colla S, Bonomini S, Hojden M, Rizzoli V and Bonati A: Downmodulation of ERK protein kinase activity inhibits VEGF secretion by human myeloma cells and myeloma-induced angiogenesis. Leukemia 18: 628-635, 2004.

29. Menu E, Kooijman R, Van Valckenborgh E, Asosingh K, Bakkus M, Van Camp B and Vanderkerken K: Specific roles for the PI3K and the MEK-ERK pathway in IGF-1-stimulated chemotaxis, VEGF secretion and proliferation of multiple myeloma cells: Study in the 5T33MM model. Br J Cancer 90: 1076-1083, 2004. 